\title{
Characterizing Extreme Points as Basic Feasible Solutions in Infinite Linear Programs
}

\author{
Archis Ghate ${ }^{a}$ and Robert L. Smith b \\ andustrial Engineering, University of Washington, Box 352650, Seattle, Washington, 98195, USA, \\ archis@u.washington.edu. \\ b'Industrial and Operations Engineering, University of Michigan, Ann Arbor, Michigan, 48109, USA, \\ rlsmith@umich.edu. \\ Unlike in finite dimensions, a basic feasible solution characterization of extreme points does not hold in count- \\ ably infinite linear programs. We develop regularity conditions under which such a characterization is possible. \\ Applications to infinite network flow problems and non-stationary Markov decision processes are presented.
}

\section{Introduction}

We consider linear programs with countably many equality constraints and variables, i.e., Countably Infinite Linear Programs (CILPs). CILPs subsume infinite network flow problems [4] and non-stationary infinite horizon deterministic and stochastic dynamic programs [3].

Recall that a vector $x$ in a convex subset $S$ of a vector space is called an extreme point of $S$ if it cannot be expressed as a strict convex combination of two distinct vectors in $S$ [1]. In standard form finite-dimensional linear programs (henceforth LPs), a feasible solution is an extreme point if and only if it can be obtained as the unique solution to the system of equations derived from the equality constraints by setting a subset of variables to zero (Theorem 2.3 in [2]). This subset consists of the so-called non-basic variables while the remaining variables are termed basic. This has proven critical in LPs as for example in designing pivot operations that exchange a variable from the non-basic set with a variable in the basic set, leading to the Simplex method.

Unfortunately, although optimal solutions of CILPs often occur at extreme points, Example 1 illustrates that the above finite dimensional characterization of extreme points does not extend to CILPs heretofore limiting research in this area.
Example 1 (modified from [4]) Consider the following CILP

$$
\begin{aligned}
x_{1}+x_{2} & =2, x_{1}-x_{3}=\frac{1}{4}, x_{2}-x_{4}=\frac{1}{4} \\
x_{3}-x_{5} & =\frac{1}{8}, x_{4}-x_{6}=\frac{1}{8}, x_{5}-x_{7}=\frac{1}{16} \\
\ldots & \\
x_{i} & \geq 0, i=1,2 \ldots
\end{aligned}
$$

One can check that vectors $u=$ $(3 / 2,1 / 2,5 / 4,1 / 4,9 / 8,1 / 8, \ldots)^{T}$ and $v=$ $(1 / 2,3 / 2,1 / 4,5 / 4,1 / 8,9 / 8, \ldots)^{T}$ are extreme points and yet clearly do not provide a unique solution to the equality constraints.

Such pathological examples are common in infinite-dimensional linear programs in abstract vector spaces [1]. The fundamental hurdle is that for a non-negative point $x$ in the variable space of the linear program, the subspace $\beta(x)$ of points whose support set is contained in that of $x$ may be strictly larger (with respect to inclusion) than the subspace $B(x)$ of bi-directions at $x$ feasible with respect to non-negativity [1]. The former is intimately related to the concept of a basic solution whereas the latter an extreme point.

We attempt to reduce this crucial gap for the case of CILPs in this paper as follows : (i) Basic and Basic Feasible Solutions (BFS) are formalized for CILPs showing that a BFS is an extreme point but the converse is not true. 
"Strictly Positive Support" (SPS) - a regularity condition under which a CILP extreme point is its BFS is introduced. SPS requires that the infimum of strictly positive components of an extreme point be strictly positive and is thus easy to check. (iii) The notion of a basic sequence, which extends the finite dimensional concept of linear independence is presented, and its relation to extreme points and basic solutions is investigated (iii) Applications from infinite network flow problems and non-stationary infinite horizon Markov decision processes are discussed in the last section.

\section{Characterization of extreme points}

Recall that $R^{\infty}$ is the space of sequences $\left\{x_{i}\right\}_{i=1}^{\infty}$ of real numbers. Constraints in standard form CILPs are written as:

$$
\begin{aligned}
& \sum_{j=1}^{\infty} a_{i j} x_{j}=b_{i}, \quad i=1,2, \ldots \\
& x_{i} \geq 0, \quad i=1,2, \ldots
\end{aligned}
$$

where $a_{i j}$ are real entries of a doubly infinite matrix $A$, and $b, x \in R^{\infty}$. We assume that the infinite sums are well-defined and finite. This holds for example when $x$ belongs to the space of bounded sequences $l_{\infty}=\left\{x \in R^{\infty}: \sup _{i}\left|x_{i}\right|<\right.$ $\infty\}$ and each row $a_{i} \equiv\left\{a_{i j}\right\}_{j=1}^{\infty}$ of matrix $A$ is in $l_{1}$, the space of absolutely summable sequences $\left\{x \in R^{\infty}:\left|x_{1}\right|+\left|x_{2}\right|+\ldots<\infty\right\}$. It also holds when $x \in l_{1}$ and $a_{i} \in l_{\infty}$, and when $x \in R^{\infty}$ and $A$ has finitely supported rows, i.e., for each $i$ the set $J(i)=\left\{j: a_{i j} \neq 0\right\}$ is finite. For concreteness, we focus on the case where $x \in l_{\infty}$, and each row as well as column of $A$ is in $l_{1}$. We now recall standard definitions and some notation.

Definition 2.1. [1] A vector $x$ feasible to (1-2) is said to be an extreme point if it cannot be written as $x=\lambda y+(1-\lambda) z$ where $\lambda \in(0,1)$ and $y \neq z$ are feasible to (1-2).

Definition 2.2. [1] For $0 \leq x \in l_{\infty}$, the subspace $B(x)$ is defined as $\left\{u \in l_{\infty} \mid x+\lambda u \geq 0, x-\lambda u \geq\right.$ 0 for some $\lambda>0\}$.
Note that $N(A)$, the null space of $A$, is given by the set $\left\{u \in l_{\infty}: \sum_{j=1}^{\infty} a_{i j} u_{j}=0, \forall i\right\}$. This leads to an algebraic characterization of extreme points.

Proposition 2.3. [1] A vector $x$ feasible to (1-2) is an extreme point if and only if $N(A) \cap B(x)=$ $\{0\}$.

Let $S(x)$ denote the support of $x$, i.e., the sequence (or set) of indices $i$ such that $x_{i} \neq 0$. The next two definitions are motivated by their finitedimensional counterparts $[1,2]$.

Definition 2.4. For any $x \in l_{\infty}, \beta(x)$ is the subspace $\left\{u \in l_{\infty} \mid u_{i}=0 \quad \forall i \notin S(x)\right\}$.

Definition 2.5. We say that $x \in l_{\infty}$ is a basic solution if it is the unique solution of (1) in $\beta(x)$. A basic solution that satisfies (2) is called a BFS.

Proposition 2.6. If $x$ is a BFS then it is an extreme point.

Proof. Suppose $x$ is not an extreme point. Then $\exists$ two distinct $y, z$ feasible to $(1-2)$ and a $\lambda \in(0,1)$ such that $x=\lambda y+(1-\lambda) z . \quad x_{i}=0$ implies $y_{i}=z_{i}=0$, since $y_{i} \geq 0, z_{i} \geq 0 \forall i$. Hence, $y, z \in \beta(x)$. Since, $y, z$ satisfy (1), this contradicts uniqueness of $x$, i.e., $x$ is not basic.

Example 1 above shows that converse of Proposition 2.6 is not true. We explore conditions under which the converse holds (Proposition 2.9 and Corollary 2.12). We first dispose of the trivial situation when $x=0$ is feasible to (1). In that case, $S(x)=\emptyset$ and $\beta(x)=\{0\}$. Thus, if $x=0$ is an extreme point, then it is trivially the unique solution in $\beta(x)$ to (1). Hence we focus on vectors $x \neq 0$, i.e., $S(x) \neq \emptyset$, in the sequel.

Proposition 2.7. Suppose $x \in l_{\infty}$ satisfies (1). Then $x$ is a basic solution if and only if $N(A) \cap$ $\beta(x)=\{0\}$.

Proof. Suppose $x$ is not basic. Then $\exists$ a $y \in$ $\beta(x), y \neq x$ such that $\sum_{j \in S(x)} a_{i j} y_{j}=b_{i} \forall i$. Since $x$ satisfies (1), $\sum_{j \in S(x)} a_{i j} x_{j}=b_{i}$ for all $i$. Thus $\sum_{j \in S(x)} a_{i j}\left(y_{j}-x_{j}\right)=0$ for all $i$. This implies that 
$\sum_{j} a_{i j}\left(x_{j}-y_{j}\right)=0$ for all $i$ because $x_{j}=y_{j}=0$ for $j \notin S(x)$. Thus $0 \neq x-y \in N(A)$ and note that $x-y \in \beta(x)$. This contradicts $N(A) \cap \beta(x)=\{0\}$.

Suppose $\exists$ a $y \neq 0$ such that $y \in N(A) \cap \beta(x)$. Then $x+y \in \beta(x)$ and $\sum_{j \in S(x)} a_{i j}\left(x_{j}+y_{j}\right)=b_{i}$ for all $i$. The latter follows since $x$ satisfies (1) and $\sum_{j \in S(x)} a_{i j} y_{j}=0$ for all $i$ as $y_{j}=0$ for $j \notin S(x)$ and $y \in N(A)$. This implies that $x$ is not basic.

Propositions 2.3, 2.6, and 2.9 shed light on a fundamental reason why an extreme point may not be a BFS - subspaces $\beta(x)$ and $B(x)$ are not necessarily equal in CILPs as illustrated in this example.

Example 2 (from [1]) Consider $x \in l_{\infty}, x \geq 0$ such that

$$
x_{1}=1, x_{i}-x_{i+1}=\frac{1}{2^{i}}, i=1,2, \ldots
$$

For $x^{*}=(1,1 / 2,1 / 4, \ldots), \beta\left(x^{*}\right)=l_{\infty}$, but $B\left(x^{*}\right)$ is the set of sequences with finitely many nonzeros. Therefore $B\left(x^{*}\right) \subset \beta\left(x^{*}\right)$.

More generally,

Lemma 2.8. $B(x) \subseteq \beta(x)$ for all $0 \leq x \in l_{\infty}$.

Proof. Let $u \in B(x)$. Then $\exists$ a $\lambda>0$ such that $x+\lambda u \geq 0$ and $x-\lambda u \geq 0$. Thus, each component of $x+\lambda u$ and $x-\lambda u$ is non-negative. Consider any $i$ with $x_{i}=0$. Then $\lambda u_{i} \geq 0$ and $-\lambda u_{i} \geq 0$. Since $\lambda>0, u_{i} \geq 0$ and $-u_{i} \geq 0$. Therefore, $u_{i}=0$ for $i \notin S(x)$. Thus, $u \in \beta(x)$.

The above discussion leads to the following converse of Proposition 2.6.

Proposition 2.9. Suppose $x$ is an extreme point with $\beta(x) \subseteq B(x)$. Then $x$ is a BFS.

We now provide a condition that is easy to check and is equivalent to $\beta(x) \subseteq B(x)$.

Definition 2.10. $0 \leq x \in l_{\infty}$ has Strictly Positive Support (SPS) if $\left(\inf _{i \in S(x)} x_{i}\right)>0$.

Note that when $x$ has integer components, it has SPS. Similarly when $S(x)$ is finite.
Lemma 2.11. Let $0 \leq x \in l_{\infty}$. Then $x$ has $S P S$ if and only if $\beta(x) \subseteq B(x)$, i.e., $\beta(x)=B(x)$.

Proof. Suppose $\beta(x) \subseteq B(x)$. Define $u \in l_{\infty}$ as follows: $u_{i}=0$ if $x_{i}=0$, and $u_{i}=1$ if $x_{i} \neq 0 . u$ is in $\beta(x)$ and hence in $B(x)$. Hence, $\exists$ a $\lambda>0$ so that $x-\lambda u>0$. Thus, $x_{i}>\lambda \forall i \in S(x)$. Thus, $x$ has strictly positive support.

Now suppose $x$ has strictly positive support and $\left(\inf _{i \in S(x)} x_{i}\right)=\delta>0$. Let $0 \neq u \in \beta(x)$ and let $\|u\|_{\infty}=C(u)>0$. Then it is easy to check that $u \in B(x)$ by choosing $\lambda=\delta / 2 C(u)$. Therefore, $\beta(x) \subseteq B(x)$.

Corollary 2.12. Suppose $x$ is an extreme point with SPS. Then $x$ is a BFS.

An independent proof of Corollary 2.12 is also possible from first principles by perturbation arguments as in LPs [2]. Note that SPS is not necessary for an extreme point to be a BFS. For instance, in Example 2, feasible solution $x^{*}=(1,1 / 2,1 / 4, \ldots)$ does not have SPS, but is an extreme point as well as a BFS (because $N(A)=\{0\}$ implying $N(A) \cap \beta(x)=\{0\})$.

Recall that in LPs, columns of the constraint matrix corresponding to the support of an extreme point are linearly independent (LI). In order to extend this notion to CILPs, let $A_{j}$ denote the $j$ th column of $A$. Using the topology of componentwise convergence on $R^{\infty}, \sum_{j=1}^{\infty} x_{j} A_{j}=b$ provides an equivalent way to write (1). We define $A_{S(x)}$ as the sequence $\left\{A_{i}\right\}_{i \in S(x)}$, and say that $A_{S(x)}$ is the sequence of columns of $A$ used by $x . A_{S(x)}$ is said to be LI when every finite subsequence of $A_{S(x)}$ is LI. To see that LI of $A_{S(x)}$ is not sufficient for a feasible $x$ to be an extreme point, consider the vector $w=(1,1,3 / 4,3 / 4,5 / 8,5 / 8, \ldots)^{T}$ in Example 1. $A_{S(w)}=A$ is LI but $w$ is not an extreme point since $w=(u+v) / 2$. On the other hand we have

Proposition 2.13. Suppose $x$ is an extreme point. Then $A_{S(x)}$ is LI.

Proof. Suppose $A_{S(x)}$ is not LI. Then $\exists$ some finite subsequences $I$ of $S(x)$ such that the set of columns $A_{I}=\left\{A_{j}: j \in I\right\}$ is linearly dependent. 
Hence $\exists$ real numbers $\{\alpha\}_{I}$ not all zero and indexed by elements of $I$ such that $\sum_{j \in I} \alpha_{j} A_{j}=0$. Now define a vector $x(\theta) \in l_{\infty}$ as follows: $x_{j}(\theta)=$ $x_{j}+\theta \alpha_{j}$ for $j \in I, x_{j}(\theta)=x_{j}$ for $j \notin I$, and $x_{j}(\theta)=0$ for $j \notin S(x)$. Clearly, $\sum_{j=1}^{\infty} x_{j}(\theta) A_{j}$ equals $\sum_{j \in S(x)} x_{j} A_{j}+\theta \sum_{j \in I} \alpha_{j} A_{j}=b$ for all real numbers $\theta$. Since $I$ is a finite set, we can ensure $x(\epsilon)$ and $x(-\epsilon)$ are feasible by choosing $\epsilon>0$ small enough. Moreover, $x=(x(\epsilon)+x(-\epsilon)) / 2$ implying $x$ is not an extreme point.

We now develop the notion of a basic sequence and argue that it is sufficient for an extreme point.

Definition 2.14. For $x \in l_{\infty}$, the set of all $y \in R^{\infty}$ for which $\exists a u \in \beta(x)$ such that $y=\sum_{j \in S(x)} u_{j} A_{j}$ will be called the countable span of $A_{S(x)}$ and denoted $\left[A_{S(x)}\right]$.

Definition 2.15. We say that $A_{S(x)}$ is a basic sequence (for $\left[A_{S(x)}\right]$ ) when every $y \in\left[A_{S(x)}\right]$ can be uniquely written as $y=\sum_{j \in S(x)} u_{j} A_{j}$ using some $u \in \beta(x)$.

If $A_{S(x)}$ is a basic sequence then $A_{S(x)}$ is LI. In Example 1, columns of $A$ are LI, however, they do not form a basic sequence since $b \in[A]$ and $u$ and $v$ both use all columns of $A$ to construct $b$, implying that the notion of LI is weaker than that of a basic sequence.

It is easy to see that an $x \in l_{\infty}$ that satisfies (1) is a basic solution if and only if $A_{S(x)}$ is a basic sequence. This implies that if $x$ is feasible to (1)-(2) and $A_{S(x)}$ is a basic sequence then $x$ is an extreme point. Moreover, if $x$ is an extreme point and $\beta(x) \subseteq B(x)$, i.e., $x$ has SPS, then $A_{S(x)}$ is a basic sequence.

\section{Applications}

Results in Section 2 are now applied to infinite network flow and Markov decision problems.

\subsection{Infinite network flow problems}

Let $G=(N, E)$ be a directed network consisting of a countable set $N=\{1,2,3, \ldots\}$ of nodes and a set $E \subseteq N \times N$ of directed arcs. For each node $i \in N$, we define $I(i)$ and $O(i)$ as the sets of incoming and outgoing arcs at node $i$ respectively. We assume that in degree and out degree of each node is finite, i.e., $|I(i)|<\infty$ and $|O(i)|<\infty$ for each $i \in N$ and further that the total degree of any node is uniformly bounded above. For each node $i \in N$, let $d(i) \in R$ be the net demand at node $i$. Then the flow balance constraints of a typical infinite network flow (henceforth INF) problem are written as follows:

$$
\begin{array}{r}
\sum_{(j, i) \in I(i)} x(j, i)-\sum_{(i, j) \in O(i)} x(i, j)=d(i) \forall i \in N \\
x(i, j) \geq 0 \quad \forall(i, j) \in E .
\end{array}
$$

We restrict our attention to the case where the flow in every arc is bounded, i.e., $x \in l_{\infty}$. A flow vector $x \in l_{\infty}$ is said to be feasible for the above problem if it satisfies the demand as well as the non-negativity constraints. Romeijn et al. [4] showed that extreme points of the set of feasible flows can be characterized using the concepts of paths and cycles in graph $G$. We now show that their results are special cases of our work.

Following Romeijn et al. [4] we define a path in graph $G$ to be a (finite or infinite) sequence of nodes $i_{1}, i_{2}, i_{3}, \ldots$ such that no node is repeated in the sequence and for each node $i_{j}$, either $\left(i_{j}, i_{j+1}\right) \in A$ (forward arc) or $\left(i_{j+1}, i_{j}\right) \in A$ (backward arc). Note that this path is not directed. A cycle in graph $G$ is defined as a finite path $i_{1}, i_{2}, \ldots, i_{k}$ with an additional arc $\left(i_{1}, i_{k}\right)$ or $\left(i_{k}, i_{1}\right)$. We say that two nodes $i, j \in N$ are connected if there exists a finite path $i_{1}, i_{2}, \ldots, i_{k}$ in $G$ with $i_{1}=i$ and $i_{k}=j$. Graph $G$ is connected if every pair of nodes in $G$ is connected. We assume that $G$ is connected. For a feasible flow $x$, we define $E(x)=\{(i, j) \in E: x(i, j)>0\}$ as the set of free arcs, and $G(x)=(N, E(x))$ as the free arc graph. Observe that the set of free $\operatorname{arcs} E(x)$ is a special case of support $S(x)$ defined earlier for CILPs. Moreover, the set $\beta(x)$ now becomes the set of flows $u$ such that $u(i, j)=0$ for all arcs $(i, j) \notin E(x)$.

Lemma 3.1. Let flow $x$ be feasible to INF. Then $x$ is a BFS (in the sense of Definition 2.5) if and only if (i) $G(x)$ has no cycles and (ii) for any 
node $i \in N$ there exists at most one infinite path $i, i_{1}, i_{2}, \ldots$ in $G(x)$.

Proof. Suppose (i) does not hold and let $i_{1}, \ldots, i_{k}, i_{1}$ be a cycle in $G(x)$ with $F \subseteq E(x)$ and $B \subseteq E(x)$ the sets of forward and backward arcs respectively in this cycle. Construct a new flow $y$ in $\beta(x)$ by setting $y(i, j)=x(i, j)$ for $(i, j) \in\{E(x) \backslash[F \cup B]\}, y(i, j)=x(i, j)+1$ for $(i, j) \in F, y(i, j)=x(i, j)-1$ for $(i, j) \in B$ and $y(i, j)=0$ for $(i, j) \notin E(x)$. Then $y$ is feasible to equality constraints contradicting uniqueness of $x$. Now suppose (ii) does not hold. Then $\exists$ a node $i^{*} \in N$ such that there are at least two paths $i^{*}, i_{1}, i_{2}, \ldots$ and $i^{*}, j_{1}, j_{2}, \ldots$ to infinity in $G(x)$. Let $I_{1} \subseteq E(x)$ and $I_{2} \subseteq E(x)$ denote the sets of arcs in these two paths respectively. Let $F_{1} \in I_{1}, B_{1} \in I_{1}, F_{2} \in I_{2}, B_{2} \in I_{2}$ denote the forward and backward arcs in these two paths respectively. Construct a new flow $y$ in $\beta(x)$ by setting $y(i, j)=x(i, j)$ for $(i, j) \in\left\{E(x) \backslash\left[I_{1} \cup I_{2}\right]\right\}$, $y(i, j)=x(i, j)+1$ for $(i, j) \in F_{1}, y(i, j)=$ $x(i, j)-1$ for $(i, j) \in B_{1}, y(i, j)=x(i, j)-1$ for $(i, j) \in F_{2}, y(i, j)=x(i, j)+1$ for $(i, j) \in B_{2}$, and $y(i, j)=0$ for $(i, j) \notin E(x)$. Then $y$ is feasible to equality constraints contradicting uniqueness of $x$. This proves the "only if" part.

For the "if" part, suppose $x$ and $y$ are two distinct solutions in $\beta(x)$ to the equality constraints. Then $x-y \neq 0$ is feasible to network $G(x)$ with with zero demand or supply on every node. Hence, either (i) or (ii) must not hold.

A basic solution for network flow problems was defined in [4] as a flow that satisfies conditions (i) and (ii) in Lemma 3.1. The Lemma shows that this concrete definition in [4] is a special case of our general notion of a basic solution. This Lemma and the results of Section 2 lead to a characterization of extreme points of INF as in [4]. Specifically, if $x$ is feasible to INF such that (i) $G(x)$ has no cycles and (ii) For any node $i \in N$ there exists at most one infinite path $i, i_{1}, i_{2}, \ldots$ in $G(x)$ then $x$ is an extreme point of INF. If $x$ has SPS and is an extreme point of INF then (i) $G(X)$ has no cycles and (ii) for any node $i \in N$ there exists at most one infinite path $i_{1}, i_{2}, \ldots \in G(X)$. Finally, as in finite network flow problems [2], if $x$ is an extreme point of INF then $G(x)$ has no cycles.

\subsection{Markov decision processes}

Consider a dynamic system observed by a decision maker at the beginning of periods $n=$ $1,2, \ldots$ to be in a period-indexed state $s_{n}$ from a finite set $S_{n}$. The initial state is $s_{1}$. The decision maker chooses an action $a_{n}$ from a finite set $A_{n}\left(s_{n}\right) \neq \emptyset$ of feasible actions in state $s_{n}$. Given that an action $a_{n} \in A_{n}\left(s_{n}\right)$ was chosen in state $s_{n}$, the system transforms into state $s_{n+1} \in S_{n+1}$ with probability $p_{n}\left(s_{n+1} \mid s_{n}, a_{n}\right)$, incurring nonnegative $\operatorname{cost} c_{n}\left(s_{n}, a_{n} ; s_{n+1}\right) \leq c<\infty$. This procedure continues ad infinitum. The term Markovian policy denotes a rule that dictates choice of action in every state (irrespective of the earlier states visited or actions taken). The goal is to find a Markovian policy that minimizes total infinite horizon discounted expected cost when the discount factor is $0<\alpha<1$.

Note that the state-space $S=\left\{S_{1} \cup S_{2} \ldots\right\}$ and the action-space $\left(\bigcup_{n=1,2, \ldots}\left(\bigcup_{s_{n} \in S_{n}} A_{n}\left(s_{n}\right)\right)\right)$ are both countable unions of finite sets and therefore countable. We consider the most common situation in applications where cardinality $\left|S_{n}\right|$ is (or uniformly bounded above by) a constant integer $M$ independent of $n$. This often happens in practice, for example, when the finite sets of possible states are identical in every period in all respects other than the period-index. Then the total number of states up to period $n$ is at most linear in $n$. Similarly, we focus on the typical situation in practice where the cardinalities $\left|A_{n}\left(s_{n}\right)\right|$ are uniformly bounded above by an integer $B$ independent of $n$ and $s_{n}$.

Let $K\left(s_{n}, a_{n}\right) \subseteq S_{n+1}$ denote the set of states $s_{n+1} \in S_{n+1}$ such that $p_{n}\left(s_{n+1} \mid s_{n}, a_{n}\right)>$ 0 . Let $c_{n}\left(s_{n}, a_{n}\right)$ denote the expected cost incurred on choosing actions $a_{n} \in A_{n}\left(s_{n}\right)$ in state $s_{n} \in S_{n}$. That is, $c_{n}\left(s_{n}, a_{n}\right)=$ $\sum_{s_{n+1} \in K\left(s_{n}, a_{n}\right)} p_{n}\left(s_{n+1} \mid s_{n}, a_{n}\right) c_{n}\left(s_{n}, a_{n} ; s_{n+1}\right)$. Finally, for any state $s_{n} \in S_{n}$, let $L\left(s_{n}\right)$ denote the set of states $z_{n-1} \in S_{n-1}$ such that there exists an action $a_{n-1} \in A_{n-1}\left(z_{n-1}\right)$ with $p_{n-1}\left(s_{n} \mid z_{n-1}, a_{n-1}\right)>0$. For each $z_{n-1}$ in $L\left(s_{n}\right)$, 
we use $\Lambda\left(z_{n-1}, s_{n}\right)$ to denote the set of actions $a_{n-1} \in A_{n-1}\left(z_{n-1}\right)$ with $p_{n-1}\left(s_{n} \mid z_{n-1}, a_{n-1}\right)>$ 0 . Let $\left\{\delta\left(s_{n}\right)\right\}$ be a sequence of positive numbers indexed by states $s_{n} \in S_{n}$ for all periods $n$ such that $\sum_{n=1}^{\infty} \sum_{s_{n} \in S_{n}} \delta\left(s_{n}\right)<\infty$. Then the infinite horizon decision problem is equivalent to solving a CILP whose constraints are given by (see [3]):

$$
\begin{array}{r}
\sum_{a \in A_{n}(s)} x(s, a)- \\
\sum_{z \in L\left(s_{n}\right)} \sum_{b \in \Lambda(z, s)} \alpha p_{n-1}(s \mid z, b) x(z, b) \\
=\delta(s), \\
\forall s \in S_{n}, n=1,2, \ldots \\
x(s, a) \geq 0, \forall s \in S_{n}, a \in A_{n}(s), n=1,2, \ldots
\end{array}
$$

Simple algebra shows that the decision vector $x$ belongs to $l_{\infty}$, each row as well as column of the equality constraint matrix is in $l_{1}$, and the above system has a feasible solution. Also, in every feasible solution $x$, for every $n$ and every $s \in S_{n}, x(s, a)>0$ for at least one $a \in A_{n}(s)$. Following [3], we define the probability of choosing an action $a_{n} \in A_{n}\left(s_{n}\right)$ in state $s_{n} \in S_{n}$ as $P_{s_{n}}\left(a_{n}\right)=x\left(s_{n}, a_{n}\right) / \sum_{b_{n} \in A_{n}\left(s_{n}\right)} x\left(s_{n}, b_{n}\right)$, for a feasible $x$. A deterministic policy is a policy where $\forall n$ and $\forall s_{n} \in S_{n}, P_{s_{n}}\left(a_{n}\right)$ is 1 for exactly one $a_{n} \in A_{n}\left(s_{n}\right)$ and 0 for all other feasible actions in $s_{n}$. That is, deterministic policies are characterized by feasible solutions $x$ where $\forall n$ and $\forall s \in S_{n}, x(s, a)>0$ for exactly one $a \in A_{n}(s)$ and $x(s, a)=0$ for all other $a \in A_{n}(s)$.

Lemma 3.2. Let $x$ be feasible to PMDP. Then $x$ is a BFS if and only if it defines a deterministic policy.

Proof. (Sketch) Suppose $x$ defines a deterministic policy. For simplicity, we number the states $1,2, \ldots$ such that if $i<j$ then $i \in S_{m}$ and $j \in S_{n}$ for some $m \leq n$. In particular, for every state $i$, the feasible action $a$ for which $x(i, a)>0$ is denoted $a_{i}$. Every $y$ in $\beta(x)$ is such that $x(i, a)=0$ $\Rightarrow y(i, a)=0$. If such a $y$ satisfies the equality constraints, then $y\left(1, a_{1}\right)=\delta(1)=x\left(1, a_{1}\right)$, $y\left(2, a_{2}\right)=\delta(2)+\alpha p\left(2 \mid 1, a_{1}\right) \delta(1)=x\left(2, a_{2}\right)$. Con- tinuing inductively, we see that $y=x$, i.e., $x$ is a BFS.

Suppose $x$ defines a randomized policy, i.e., there exist states $s$ for which $x(s, a)>0$ for more than one feasible action $a$. Then one can construct a solution $y$ in $\beta(x)$ distinct from $x$ that satisfies the equality constraints (this can be achieved for example by decreasing $x(s, a)$ to zero for all feasible actions $a$ except for one for each state $s$ inductively to ensure that equality constraints are satisfied). Hence $x$ is not a basic solution.

Lemma 3.2 and Proposition 2.6 imply that if a feasible $x$ defines a deterministic policy then it is an extreme point. Interestingly, the converse is also true in spite of the fact that feasible solutions do not have SPS (providing another example that SPS is not necessary for Corollary 2.12), however it requires a more direct proof that we omit here.

\section{Acknowledgments}

Research supported in part by the NSF under grant DMI-0322114. The first author thanks the University of Washington for summer support.

\section{REFERENCES}

1. Anderson, E. J., and Nash, P., Linear Programming in infinite-dimensional spaces: Theory and applications, John Wiley and Sons, Chichester, Great Britain, 1987.

2. Bertsimas, D., and Tsitsiklis, J. N., Introduction to linear optimization, Athena Scientific, Belmont, Massachusetts, USA, 1997.

3. Puterman, M. L., Markov decision processes : Discrete stochastic dynamic programming, John Wiley and Sons, New York, 1994.

4. Romeijn, H. E., Sharma, D., and Smith, R. L., Extreme point solutions for infinite network flow problems, Networks, 48 (4), 209$222,2006$. 